\title{
A Retrospective Study One-Year Survey from COVID-19: Three Waves and Three Patterns in Italy
}

\author{
Olga Kolesova1,3), Flaminia Tommasetti2,3), Paola Cerini3), Laura Cardarelli4), \\ Laura Marullo5), Fulvio Cantore6), Elena Zocca7), Maria Loredana Frassanito3), \\ Maria Teresa Quarantelli5), Massimo Pieri3,8), Graziella Calugi ${ }^{3,4,5,6,7)}$ \\ 1)Department of Public Health and Infectious Diseases, University of Rome "La Sapienza”, Rome, Italy \\ ${ }^{2)}$ Department of Life Sciences and Biotechnology, University of Ferrara, Ferrara, Italy \\ 3)Lifebrain srl; Viale Roma 190/A, Guidonia Montecelio, Rome, Italy \\ 4)RDI srl, Via del Santo, 147, Limena, Padua, Italy \\ 5)Lifebrain Campania, Via Nicola Bruni Grimaldi, 95, Nocera Inferiore, Salerno, Italy \\ 6) Lifebrain Puglia, Viale Virgilio, 97/A, Taranto, Italy \\ 7)Lifebrain Veneto, Via Marconi, 30, Piombino Dese, Padua, Italy \\ 8)Department of Experimental Medicine, University of Tor Vergata, Rome, Italy
}

\section{ABSTRACT}

Background: Since December 2019 an unprecedented coronavirus pandemic (COVID-19) in Italy and in all the world, has been spreading. This study is a retrospective overview of Italian population in one year of COVID-19. This study aimed to assess the changes of COVID-19 distribution during the first, second and third waves throughout Italy.

Subjects dan Method: This was a retrospective study carried out in all Italian regions from symptomatic or COVID-19 high-risk exposure patients. 41,135,655 nasopharyngeal swabs samples were obtained and analyzed between man and woman in five age categories. The dependent variables were positivity rate and collection period. The independent variables were age and gender. The swabs were processed by qRT-PCR technique. The data were extrapolated by QLink software and evaluated using chi-square test statistical analysis.

Results: The paper presents one year overview epidemiological data of three COVID-19 waves all over Italy. It was considered a number of 122,853 out of 41,135,655 (10.8\%) positive patients in one year of pandemic. The article briefly reports three epidemic outbreaks caused by the SARS-CoV-2 with positivity rate of $5.13 \%, 18.07 \%$ and $14.02 \%$, respectively. It was evaluated how the patients average age has shifted from elders towards younger, observing a high percentage (45.13\%) in over 65 group in the first wave despite the $0-8$ (1.00\%) and $8-18$ age groups (2.66\%). Contrary, the number of positive cases in $0-8$ and 8-18 age groups increased in second (1.06\% and $2.66 \%)$ and third waves (2.06\% and $4.39 \%)$.

Conclusion: It presents the current epidemic situation associated with the novel coronavirus and outlines the main clinical features on the three waves in Italy. It was observed a shift toward a younger patient during the second and the third pandemic wave and a slight predominance of female on male in the first wave.

Keywords: COVID-19, SARS-CoV-2, epidemiology, outbreak, virology

\section{Correspondence:}

Massimo Pieri. University of Rome "Tor Vergata", Department of Experimental Medicine, Via Montpellier 1, 00133 Rome, Italy. Email: massimo.pieri@uniroma2.it. Mobile: 0774345000.

Cite this as:

Kolesova O, Tommasetti F, Cerini P, Cardarelli L, Marullo L, Cantore F, Zocca E, et al. (2021). A Retrospective Study One-Year Survey from COVID-19: Three Waves and Three Patterns in Italy. J Epidemiol Public Health. 06(04): 392-401. https://doi.org/10.26911/jepublichealth.2021.06.04.01.

(c) (7) (2) Journal of Epidemiology and Public Health is licensed under a Creative Commons Attribution-NonCommercial-ShareAlike 4.o International License. 


\section{BACKGROUND}

Official information about a new outbreak of pneumonia of unknown etiology in Wuhan City, the capital of Hubei Province of China, appeared for the first time on December 31, 2019 (Xu et al., 2020). On January 3rd, 2020, this new disease was already confirmed in 44 patients. All of them were adult residents of Wuhan city linked with the local animal and seafood market. These patients developed symptoms between December 12 and 29, 2019. The incubation period lasted from 2 to 14 days and the febrile period from 10 to 14 days (WHO, 2020a). In early January 2020, Shanghai Public Health Clinical Center established the complete genomic sequence of this pneumonia's causative agent, a new coronavirus strain, temporarily named 2019 Novel coronavirus (2019-nCoV), which, according to World Health Organization (WHO) experts, was not previously identified (Xu et al., 2020). Later on February 2020, the new coronavirus infection was named COVID-2019 (Corona Virus Disease 2019), caused by SARS-CoV-2 (Tai et al., 2020).

Starting from January 21, 2020, WHO publishes daily Situation reports of the Emergency Committee under the International Health Regulations on pneumonia caused by SARS-CoV-2, containing information on the number of confirmed cases and deaths risk level plus infection control guidelines and other relevant information (Salzberger et al., 2020).

On February 21st, 2020 the first Italian patient with COVID-19 (a 38-yearold man) was hospitalized at Codogno Hospital, Lodi, in northern Italy (Romagnani et al., 2020). The virus spread firstly in Europe, then all around the world, becoming global plague. On 11th March 2020, WHO declared the emergency for global pandemic by SARS-CoV-2 (WHO, 2020b).
Since then, the contagious and death situation has been changing daily. According to WHO, on April 11th , 2021, the number of cases in the world was $134,308,070$ confirmed cases of COVID-19 with the maximum number of patients registered in the Americas (WHO, 2021). In terms of the number of cases of COVID-19, Italy occupies one of the leading places in the world (around 3 million cases) (Wang et al., 2020; Romagnani et al., 2020).

Many countries around the world faced COVID-19 pandemic where a first wave occurred in the spring 2020 that substantially decreased during the summer, the second massive wave emerged in the fall of 2020, and the third wave occurs again in spring 2021.

Lifebrain is the leading group in Italy for laboratory medicine for patients, and due to its laboratory network, high professionalism, and latest-generation technologies has analyzed and analyze molecular COVID-19 tests during almost all pandemic periods in most of Italian regions. Lifebrain has a massive database of molecular results in Italian waves. Anyhow, the number of cases in the first wave were limited due to restricted authorization law for diagnostic center of COVID-19.

Using original data from three following outbreaks of COVID-19, it was analyzed gender differences and age shift between three waves in Italy. The first wave was administered between March and May 2020. On March 10th 2020, the Italian government has promptly reacted to the pandemic by adopting a first set of national restrictions and recommendations, avoiding non-essential industrial productions and all the social interactions, including schools and offices (Salzberger et al., 2020; Tai et al., 2020). Despite all restriction, the second wave was administered between 
October and December 2020, the third arrived in the middle of February 2021.

This study aimed to examine the changes in patterns of COVID-19 distribution during the first, second and third waves throughout Italy. It was assessed obtained data of SARS-CoV-2 in Italy within one year of pandemic.

\section{SUBJECTS AND METHOD}

\section{Study Design}

This was a respective study to assess COVID-19 waves in Italy. The study design consisted of assessing the main patient patterns during the pandemic. The data were obtained by Qlik Sense, one innovative Business Intelligence tool. Data was collected during one year between March 2020 until the end of March 2021, covering three waves, when the number of SARS-COV-2 positive cases increased exponentially.

\section{Population and Sample}

This study used original data from the Lifebrain laboratories, one of the largest groups of private clinical structure in Italy. 41,135,655 nasopharyngeal swabs samples were obtained at the different Lifebrain network laboratories sites from either symptomatic patients or patients with high-risk exposure to COVID-19. All samples were collected in $2 \mathrm{ml}$ of viral preservation medium in Kang Jian Virus Collection and Preservation System (Jiangsu Kangjian Medical Apparatus Co., Ltd., China) and tested within 24 hours of collection.

\section{Study Variables}

The dependent variables were positivity rate and the collection period. The independent variables were age and gender.

4. Operational Definition of Variables Age: the age of patients was evaluated for each COVID-19 pandemic wave and divided in seven age range: $0-8$ years old, 8-18 years old, 18-30 years old, 30-40 years old, 40-50 years old, 50-65 years old, over 65 years old.

Gender: both male and female gender were considered, calculated for each COVID-19 wave.

Collection period: all samples were collected during first three waves of COVID-19 pandemic, first wave was administered between March and May 2020; the second wave between October and December 2020; the third between February and March 2021.

Positivity rate: samples positive for SARS-CoV-2 are characterized by a cycle threshold $(\mathrm{Ct})$ value at or below 35 cycles of the RdRp gene for Mutaplex and of ORF1ab gene for PerkinElmer.

\section{Study Instruments}

Viral RNA was extracted, starting from $200 \mu \mathrm{l}$ of swab fluid using the MGISP-960 High-throughput Automated Sample Preparation System using MGIEasy Magnetic Beads Virus DNA/RNA Extraction Kit, and Janus liquid handler, using Chemagic 360 (PerkinElmer Inc, Waltham, Massachusetts) and PerkinElmer New Coronavirus Nucleic Acid Detection Kit (Perkin Elmer Inc, Waltham, Massachusetts). The detection of SARS-CoV-2 RNA was carried out using the MutaPLEX $\AA$ Coronavirus (SARS-CoV-2) real-time RT-PCR Kit (Immundiagnostik AG, Germany) for the simultaneous in vitro detection of RNA of SARS-CoV-2 and other beta coronaviruses, extracted from biological specimens. MutaPLEX® Coronavirus (SARSCOV-2) kit (Immundiagnostik AG, Germany) detects three viral genes. The cycling conditions were: $10 \mathrm{~min}$ at $45^{\circ} \mathrm{C}, 5$ min at $95^{\circ} \mathrm{C}, 45$ cycles $\mathrm{x}\left[10 \mathrm{~s}\right.$ at $95^{\circ} \mathrm{C}, 40$ $\mathrm{s}$ at $\left.60^{\circ} \mathrm{C}\right]$.

The PerkinElmer SARS-CoV-2 RTqPCR Reagent kit (PerkinElmer Inc, Waltham, Massachusetts) was also used for the 
qualitative detection of SARS-CoV-2. The SARS-CoV-2 RT-qPCR assay uses TaqMan $^{\text {TM}}$-based real-time PCR technique to conduct in vitro transcription of SARSCoV-2 RNA, DNA amplification and fluorescence detection. The cycling conditions were: 2 min at $25{ }^{\circ} \mathrm{C}, 15 \mathrm{~min}$ at $250^{\circ} \mathrm{C}, 2$ min at $95{ }^{\circ} \mathrm{C}$ and 45 cycles $\mathrm{x}\left[3 \mathrm{~s}\right.$ at $95{ }^{\circ} \mathrm{C}$, $30 \mathrm{~s}$ at $\left.60^{\circ} \mathrm{C}\right]$.

Qlik Sense was used to extrapolate the data. It is based on the Associative InMemory Engine, that allow to explore quickly the entire Datawarehouse. The software is able to associate dynamically the data, exploring freely in any direction, not following the most common pyramidscheme. Practically, it is possible surfing data from the top side like, Company/Blood collection point, or data from the bottom, like discovery business and customer behaviour.

\section{Data analysis}

We analyzed the data of seven age groups of patients: o-8 years old, 8-18 years old, 18-30 years old, 30-40 years old, 40-50 years old, 50-65 years old, and patients over 65 years old. The rate differences in age groups, gender and waves were estimated by chi-square $\left(\chi^{2}\right)$ test.

Analyses were performed using MedCalc 19.2.0 (MedCalc Software Ltd, Ostend, Belgium).

\section{Research Ethics}

The study has been led in accordance with the Declaration of Helsinki, under the terms of relevant local legislation.

\section{RESULTS}

This study tested hypotheses on the age shift and gender differences within three COVID-19 pandemic waves. It was used a database of molecular analysis from three pandemic waves in one year with a total number of patients of 41,135,655, 122,853 (10.8\%) of which were positive cases. The main method for verifying SARS-CoV-2 is reverse transcription PCR. The age group, gender rate and total swabs number at each wave are shown in Table 1 . Also, Table 1 represents basic descriptive statistics of the original COVID-19 data obtained in Lifebrain centers all over Italy.

Table 1. Descriptive statistics at each wave in Italy, including total number, positivity rate, age and gender

\begin{tabular}{lcccccc}
\hline & \multicolumn{2}{c}{ WAVE 1 } & \multicolumn{2}{c}{ WAVE 2 } & \multicolumn{2}{c}{ WAVE 3 } \\
\hline & $\mathbf{N}$ & $\mathbf{\%}$ & $\mathbf{N}$ & $\mathbf{\%}$ & $\mathbf{N}$ & $\mathbf{\%}$ \\
\hline N. total & 54,131 & $100 \%$ & 205,997 & $100 \%$ & 73,217 & $100 \%$ \\
Positivity rate & 2,776 & $5.13 \%$ & 37,220 & $18.07 \%$ & 16,206 & $14.03 \%$ \\
o-8 years & 28 & $0.9 \%$ & 395 & $1.1 \%$ & 334 & $2.1 \%$ \\
8-18 years & 74 & $2.6 \%$ & 1,635 & $4.4 \%$ & 1,009 & $6.1 \%$ \\
18-30 years & 232 & $8.2 \%$ & 6,211 & $16.7 \%$ & 2,484 & $15.2 \%$ \\
30-40 years & 218 & $7.8 \%$ & 5,552 & $14.9 \%$ & 2,390 & $14.7 \%$ \\
40-50 years & 389 & $13.9 \%$ & 6,654 & $17.9 \%$ & 2,734 & $6.8 \%$ \\
50-65 years & 582 & $20.6 \%$ & 9,166 & $24.6 \%$ & 5,124 & $24.4 \%$ \\
Over 65 years & 1,253 & $46.1 \%$ & 7,600 & $20.4 \%$ & 3,294 & $20.7 \%$ \\
Men & 1,102 & $38.9 \%$ & 18,259 & $49.1 \%$ & 8,118 & $49.8 \%$ \\
Women & 1,674 & $61.1 \%$ & 18,961 & $50.9 \%$ & 8,088 & $50.2 \%$ \\
\hline
\end{tabular}

During the first pandemic wave, a total of 54,131 patients have used Lifebrain collection-sites to evaluate their COVID-19 status, meanwhile 205,997 and 131,907 pati- ents again at second and three waves, respectively. The summer period, including June, July, August, and September, is an intermediate period, due to the low 
number of positive cases. For this reason, it has been not considered as a wave.

The first wave of COVID-19 in Italy began in March 2020 and was distinguished by an outbreak with high positive rate of $5.13 \%(2,776$ out of 54,131$)$. Analyzing the obtained data, we found differences in the disease prevalence by age groups of population. The age statistics are reported in Figure 1. The Figure 1 shows that first pandemic wave in Italy was characterized by a severe clinical course of the disease among people over 65 years. A change of age range was observed from first wave to the next two waves; it was noted a significant $(p<0.001)$ decrease in over 65 rates between the first and subsequent two waves. There was a critically high positivity percentage of $45.13 \%(1,253$ out of 2,776 ) among people over 65 , whereas the o-8 and 8-18 age range rate was practically minimum, $1.00 \%$ (28 out of 2,776 ) and $2.66 \%$ (74 out of 2,776), respectively.

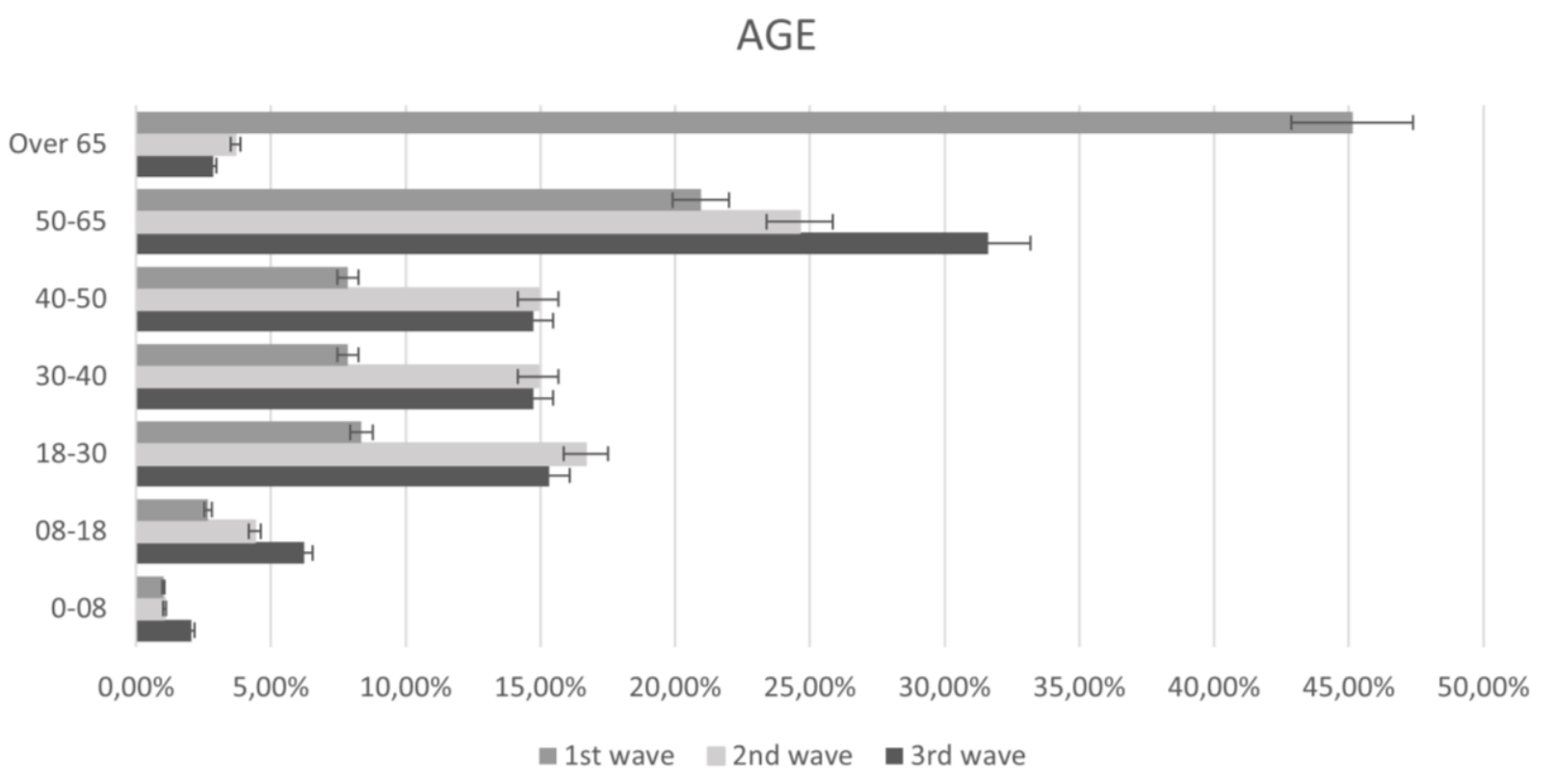

Figure 1. Shift in average age of COVID-19 patients in all Italy. The age group are related to the three waves

The second wave of the pandemic in Italy occurred in the fall of 2020, with a positivity rate of $18.07 \%$ (37,220 out of 205,997), meanwhile the third was at the beginning of 2021, with a positivity rate of $14.02 \%(16,206$ out of 115,632$)$. Compared to the pandemic first wave, the subsequent were characterized by greater involvement of different age groups, especially children $0-8$ years and adolescents aged 8-18 years. In the age group from o to 8 years, the number of positive patients doubled from the first wave to 1.06\% (395 out of 37,220 ) in the second and $2.06 \%$ (334 out of 16,206) during the third. In the age group from 8 to 18 years, an even sharper jump in the incidence rate was observed almost threefold, from $2.66 \%$ (74 out of 2,776 ) in the first wave to $4.39 \%$ $(1,635$ out of 37,220$)$ in the second and $6.22 \%$ (1,009 out of 16,206 ) during the third. However, in subsequent waves, the number of COVID-19 positive patients over 65 years significantly decreased $(\mathrm{p}<\mathrm{0.001})$. The number of patients over 65 years of age decreased to $20.42 \%$ 
$(7,600$ out of 37,220$)$ of positive cases in the second wave and $20.32 \%(3,294$ out of $16,206)$ in the third wave. In the age group between 30 and 40 , the number of positive cases during the first, the second and third waves $(7.85 \%, 218$ out of 2,776 ; $14.91 \% 5,552$ out of 37,$220 ; 14.74 \% 2,390$ out of 16,206 ) did not differ significantly from the first wave. The same trend was observed in age group between 40 and 50 (14.01\%, 389 out of 2,$776 ; 17.88 \%, 6,654$ out of 37,$220 ; 16.87 \%, 2,734$ out of $16,206)$ and in age group between 50 and $65(20.97 \%, 582$ out of 2,$776 ; 24.63 \%$,
9,166 out of 37,$220 ; 24.43 \%, 3,294$ out of 16,206).

Instead, gender differences between the last two waves were not as significant as the average age shift (Figure 2). However, during the first wave of the pandemic, it was noted a significant prevalence of morbidity among women with $61.1 \%(1,674$ out of 2,776$)$ despite of men rate $39.70 \%(1,102$ out of 2,776$)$, with $\mathrm{p}<0.01$; however, it was not recorded any statistical significance during the second and third waves. The ratio of positive cases among men and women practically was steady and comparable.

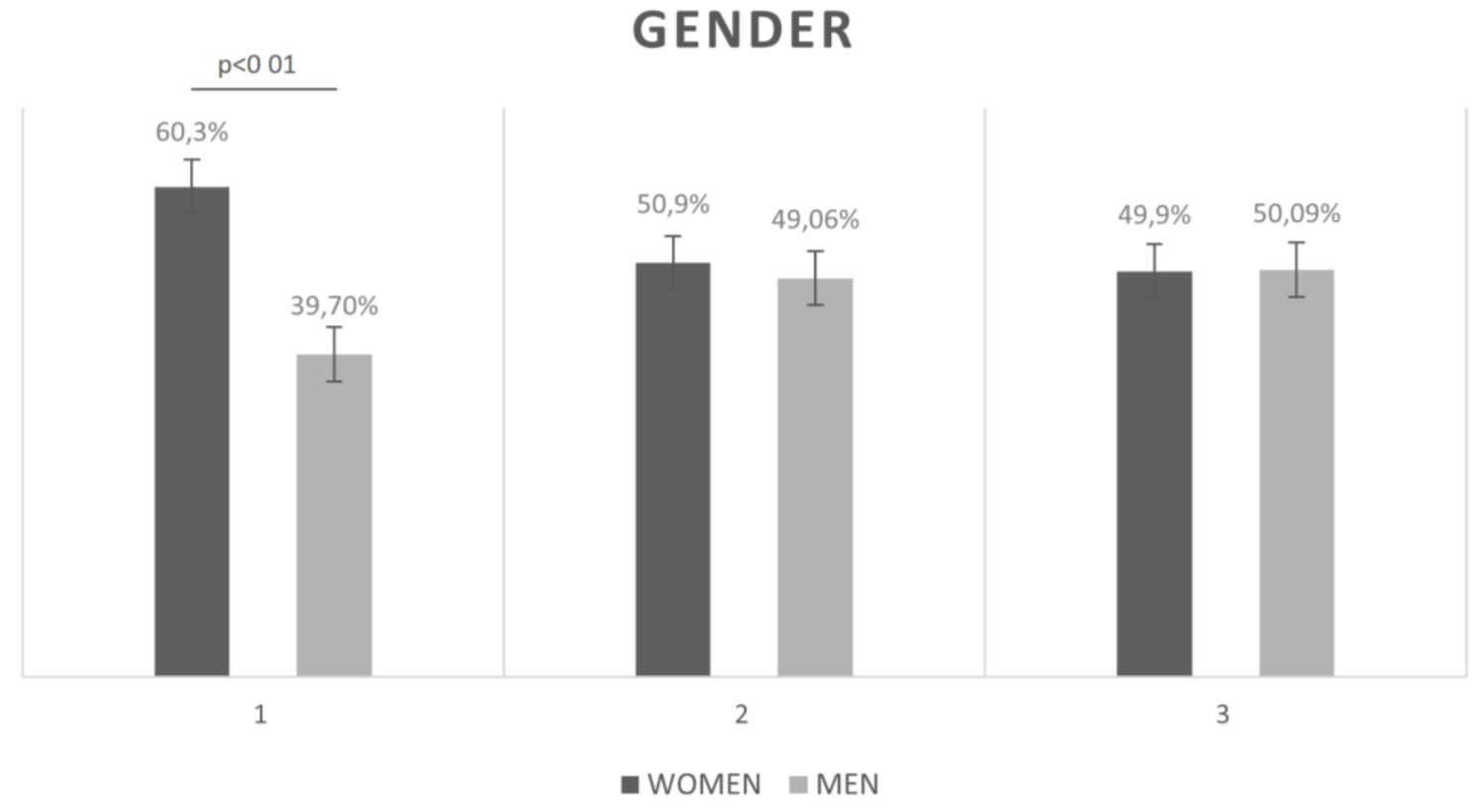

Figure 2. Male and female ratio between the three waves of COVID-19

In the first wave, gender ration has $p$ $<0.010$, instead in the second and third it was not observed no significant differences. Figure 3 shows the positivity trend through one year of COVID-19 pandemic. The trend line highlights the three waves with the positive rate and histograms represent the total of negativity-positivity (grey columns), of molecular swabs, divided by months. 


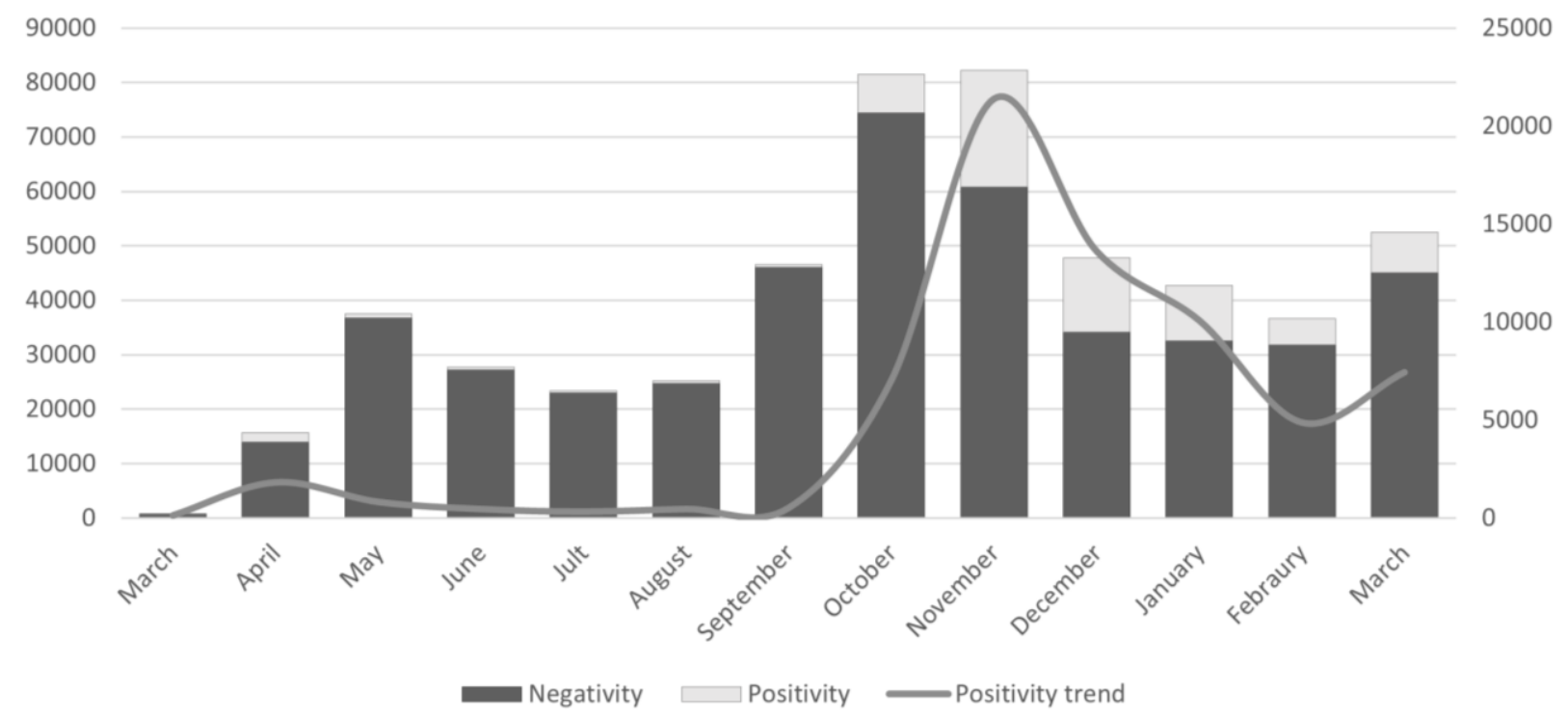

Figure 3. Positivity rate (grey line) related to the total of negativity-positivity (columns), divided for each month

\section{DISCUSSION}

In Italy and in all the world, there is an unprecedented coronavirus pandemic in modern history in terms of the number of cases and socio-economic consequences. In the end of March, more than 128 million cases of SARS-CoV-2 were registered globally, of which more than 73 million recovered, and almost 3 million people died. The most affected countries are China, USA, Italy and Spain, and the small countries of Western Europe. At the same time, governments are introducing significant, never seen before, restrictions on people movement, additional security measures, in particular, they alarm their citizens to stay at home and wear masks. A large number of employees were transferred to remote work, school holidays were extended, online education was applied.

In Italy, during the drafting of this paper, more than 3,5 million cases are officially registered, of which 2,9 million people recovered and almost 110 thousand people died. Implementing a set of measures in the country is carried out as part of COVID-19 prevention plan. Unfortunately, by the end of March 2021, cases were reported in all regions of Italy. However, despite all the efforts taken, the country is in the middle of the third wave of COVID-19 infection. The authorities of many regions are facing a dilemma: the need to reduce morbidity by introducing quarantine measures contradicts the need to reduce the negative socio-economic consequences of an equally rapidly developing economic crisis.

According to the Novel Coronavirus Pneumonia Emergency Response Epidemiology Team (NCPERET), during the first wave, the SARC-CoV-2 affected men and women almost equally, while the mortality rate among affected men was significantly higher (Lu, 2020). Besides, during the first wave, almost $78 \%$ of cases of COVID-19 were detected in persons aged 30 to 65 years inclusive (Wassenaar and Zou, 2020). While in the following outbreaks of COVID-19, there has been a shift towards infecting younger population. The intensity of the pandemic in the first, second and third waves varied 
significantly in coverage of different age groups of population.

Firstly, we evaluated the positivity rate in each age group in all three waves. The first pandemic wave was characterizeed by significant rate of elderly group contagious and a smaller number of children and adolescents contagious. In comparison with the first wave, the second wave, but mostly the third, had a shift from elder to younger patients. During the third wave, a significant increase in the number of cases among children and adolescents has been observed. This shift could be imputed by not school restriction and a moderate restarting of social relationship. At the end of third wave, the positivity rate in children $0-8$ has doubled and in adolescents 8-18 years old has tripled, in comparison with the first pandemic wave. Another possible cause of minor positivity rate increasing in second and third pandemic waves may be associated with the emergence of new mutations of the SARSCoV-2 genome.

According to scientific data, a viral infection caused by SARS-CoV-2, with varying degrees of severity of the disease (from mild to severe forms with the development of deaths), most often affects the adult population aged 30 to 79 years (86.6\%) (Monod et al., 2021). The child population is infected with the virus in 7$11 \%$ of cases and becomes sick in 1.7-2.2\% of cases (Wang and Zhang, 2020). In 90\% of cases, children carry the disease in mild, moderate and asymptomatic forms (Dong et al., 2020). Still, they can participate in the transmission of the virus (Kucharski et al., 2020), which can be extremely dangerous for people with concomitant pathology and the elderly. A significant decrease in contagious among people over 65 years could be attributed by the implementation of a set of res- trictive measures in the country, by general awareness in adult population, and by caution and self-isolation of older people.

Anyhow, the age group shifting could be due to virus adaptation, and variants arising, because, as it known, virus needs to spread and survive, and it is always looking for new hosts (Sinkovics et al., 1998).

Moreover, it was not observed gender through three pandemic waves. During the first pandemic wave, there was a slight prevalence of morbidity among men; however, the ratio of positive cases among men and women practically were steady during the second and third waves.

Due to the software, it is possible to extrapolate data in real time, with many filters, as age, as gender, as regions, automatically, without any data input. Also, the capillary network laboratories cover mostly Italian territory, and then the data obtained can be compared to the national trends of Italian Ministry of Health.

However, the current study has several limitations that should be considered during the interpretation. The data are limited to Italy, and it remains to be tested whether the age shift and gender difference between the three waved varies similarly in different countries. Also, the first wave had a small number of data, due to the Italian low restrictions on swabtesting in private facilities, as Lifebrain laboratories.

Although no gender difference was noted between positive cases, it would be interesting to observe if there is a discrepancy between age and gender during the three waves, however it was not possible extrapolate this data from the software.

Overall, our data is in agreement with Italian Ministry of Health and repre- 
sent complete picture of contagious in Italy due to capillary system.

This retrospective study provided a rare overview of population age shift and gender predominance within Italian three first COVID-19 waves. It was observed a shift toward a younger population during the second and the third pandemic wave in Italy: the number of infected children and adolescents under 18 years has more than doubled compared to the first wave. On the other hand, the number of positive cases in the age group over 65 halved.

The data from the first wave show a slight predominance of positive cases among women. However, in subsequent waves, the number of infection cases between men and women practically does not differ.

The overall data assembled on the prevalence of SARS-CoV-2 infection in one year of pandemic suggest that the virus is co-evolving in response to our defensive strategies. However, a huge scientific effort is globally focus to firstly safely coexist with the virus and then achieve held immunity, hoping the overcame of COVID-19 pandemic.

\section{CONFLICT OF INTEREST}

The authors declare that the study was conducted in the absence of any commercial or financial relationships that could be construed as a potential conflict of interest.

\section{ACKNOWLEDGMENT}

The authors would like to thank all laboratory nurses and technicians of Lifebrain laboratories for their technical support.

\section{AUTHORS CONTRIBUTION}

CG raised the initial research question, $\mathrm{CP}, \mathrm{CL}, \mathrm{ML}, \mathrm{CF}, \mathrm{ZE}, \mathrm{MTQ}$ and MLF, managed data collection, $\mathrm{KO}, \mathrm{TF}, \mathrm{PM}$ ran statistical analysis and drew tables and graphs. PM, KO and TF refined research questions, planned study design, planned and ran statistical analysis, interpreted results, and wrote up manuscript. CG and $\mathrm{PM}$ suggested issues in the discussion.

\section{FUNDING AND SPONSORSHIP} None

\section{REFERENCE}

Dong Y, Mo X, Hu Y, Qi X, Jiang F, Jiang Z, Tong $S$ (2020). Epidemiology of COVID-19 Among Children in China. Pediatrics. 145 (6). doi: 10.1542/peds. 2020-0702.

Kucharski AJ, Russell TW, Diamond C, Liu Y, EdmundS J, Funk S, Eggo RM (2020). Early dynamics of transmission and control of COVID-19: a mathematical modelling study. Lancet Infect Dis. 20 (5): 553-558. doi: 10.1016/S1473-3099(20)30144-4.

Lu H (2020). Drug treatment options for the 2019-new coronavirus (2019nCoV). Biosci Trends. 14(1):69-71. doi: 10.5582/bst.2020.01020.

Monod M, Blenkinsop A, Xi X, Hebert D, Bershan S, Tietze S, Baguelin M, et al. (2021). Age groups that sustain resurging COVID-19 epidemics in the United States. Science. 371. (6536):eabe8372. doi:10.1126/science.abe8372.

Romagnani P, Gnone G, Guzzi F, Negrini S, Guastalla A, Annunziato F, Romagnani $\mathrm{S}$, et al. (2020). The COVID-19 infection: lessons from the Italian experience. J Public Health Policy. 41: 238-244. doi: 10.1057/s41271-02000229-y.

Salzberger B, Gluck T, Ehrenstein B (2020). Successful containment of COVID-19: the WHO-Report on the COVID-19 outbreak in China. Infection. 48: 151- 
Kolesova et al./ A Retrospective Study One-Year Survey from COVID-19

153. doi: 10.1007/s15010-020-014094.

Sinkovics J, Horvath J, Horak A (1998). The origin and evolution of viruses (a review). Acta Microbiol Immunol Hung. 45: 349-390.

Tai W, He L, Zhang X, Pu J, Voronin D, Jiang S, Zhou Y, Du L (2020). Characterization of the receptor-binding domain (RBD) of 2019 novel coronavirus: implication for development of RBD protein as a viral attachment inhibitor and vaccine. Cell Mol Immunol. 17. 613-620. doi: 10.1038/s41423-020-0400-4.

Wang C, Horby PW, Hayden FG, Gao GF (2020). A novel coronavirus outbreak of global health concern. Lancet. 395: 470-473. doi: 10.1016/So140-6736(2o)30185-9.

Wang F, Zhang C (2020). What to do next to control the 2019-nCoV epidemic? Lancet. 395: 391-393. doi: 10.1016/S0140-6736(20)30300-7.

Wassenaar TM, Zou Y (2020). 2019_nCoV/SARS-CoV-2: rapid classification of betacoronaviruses and identification of Traditional Chinese Medicine as potential origin of zoonotic coronaviruses. Lett Appl Microbiol. 70: 342348. doi: 10.1111/lam.13285.
WHO (2020a). Pneumonia of unknown cause-China [Online]. Retrieved from https://www.who.int/csr/don/o5-january-2020-pneumonia-of-unkowncause-china/en/ [Accessed Jan 05, 2020].

WHO (2020b). WHO Director-General's opening remarks at the media briefing on COVID-19 - 11 March 2020 [Online]. Retrieved from https://www .who.int/director-general/speeches/ detail/who-director-general-s-opening-remarks-at-the-media-briefingon-covid-19-11-march-2020[Accessed Mar 11, 2020].

WHO (2021). WHO Coronavirus (COVID19) Dashboard, global daily report [Online]. Available: https://covid19. who.int/ [Accessed Mar 26, 2021].

Xu X, Chen P, Wang J, Feng J, Zhou H, Li X, Zhong W, Hao P (2020). Evolution of the novel coronavirus from the ongoing Wuhan outbreak and modeling of its spike protein for risk of human transmission. Sci China Life Sci. 63: 457-46o. doi: 10.1007/s11427020-1637-5. 